\title{
アファイン拘束を受ける非ホロノミックハミルトニアン システムのモデリングと受動性解析*
}

甲斐 健也 ${ }^{\dagger}$

\author{
Modeling and Passivity Analysis of Nonholonomic Hamiltonian \\ Systems with Affine Constraints*
}

Tatsuya $\mathrm{KAI}^{\dagger}$

\begin{abstract}
In this paper, we introduce and analyze Hamiltonian systems subject to nonholonomic affine constraints. We first sum up some concepts of Hamiltonian systems defined on both the phase space and the expanded phase space. Next, we derive nonholonomic Hamiltonian systems with affine constraints (NHSAC) by using a transformation and reduction on the expanded phase space. Then, passivity of the NHSAC with the control input term and the output equation is investigated. Finally, a coin on a rotating table is illustrated to confirm the results as a physical example.
\end{abstract}

\section{1. はじめに}

近年, 制御工学やロボット工学の分野において , 非ホ ロノミックシステムの研究が幅広く行われている $[8,17]$. それらの研究のほとんどは, 速度に線形な「線形拘束」 を扱っており，線形拘束を受けるシステムには以下のよ うな特徽が一般的に知られている $[1,2,8,17]$. (i) 線形近 似システムが不可制御，(ii) なめらかな非線形状態フィー ドバックで安定化不可能 . 谷のため, これらのシステム の制御は難しく，さまざまな制御則が提案されている.

一方，速度にアファインな「アファイン拘束」も存在 し，これは線形拘束を含むより広いクラスの拘束条件で ある . 初期角運動量を持つ宇宙ロボット・流れる川の上 のボート・回転盤上の球またはコインなどは , アファイ ン拘束を受けるシステムの一例である (Fig. 1). 筆者ら はこれまで，アファイン拘束に関する微分幾何学的・制 御論的な研究を行ってきた $[10-14]$. とくに $[12,13]$ では， アファイン拘束を受ける非ホロノミック運動学/動力学 システムを導出し, 非線形制御理論に基づく解析を行っ た．炎の結果，(a) 線形近似システムが可制御になる場 合がある，(b) なめらかな非線形状態フィードバックで 安定化の可能性がある，などの線形拘束の場合では見ら

* 原稿受付 2008 年 12 月 26 日

†阪大学 大学院 工学研究科 Graduate School of Engineering, Osaka University; 2-1 Yamadaoka, Suita, Osaka 565-0871, JAPAN

Key Words: nonholonomic affine constraints, Hamiltonian systems, passivity, coin on rotating table.
れなかったような新しい性質を示した . アファイン拘束 を受ける動力学システム $[13]$ はラグランジュ力学に基づ くアプローチによって導出されたものであるが, 動力学 モデリングの別方法として , ハミルトンカ学によるアプ ローチも存在する . ラグランジュ形式・ハミルトン形式 の光れ光れのシステムは, ルジャンドル変換によって互 いに等価である．しかし，ハミルトン形式は正準座標に よる正準方程式 (ハミルトニアンシステム)で記述され， 正準座標の対称性・正準変換によるシステムの特徽の不 変性などを持ち, さらにシステムのエネルギーに関する 受動性を陽に表しているなど , ラグランジュ形式よりも 優れている点が多い . van der Schaft らは , 線形拘束を 受ける非ホロノミックハミルトニアンシステムを導出し た [3]．また，Fujimoto らは線形拘束を受ける非ホロノ ミックハミルトニアンシステムに対して, 一般化正準変 換ならびに受動性や Lyapunov 関数に基づいた制御則を 提案し，有効性を示している $[5,9]$. したがって，アファ イン拘束を受けるシステムの制御でも，このようなハミ ルトン形式のアプローチが有効であると考えられる .

弚こで本論文では, ハミルトニアンシステムのアファ イン拘束を受ける場合への拡張ならびに解析を目的とし ている．まず 2.では，本論文を通して扱う非ホロノミッ クなアファイン拘束について概説する．つぎに $3 . て ゙ は ，$ 相空間ならびに拡大相空間におけるハミルトニアンシ ステムについて説明を行い, 弚れらに基づいてアファイ ン拘束を受ける非ホロノミックハミルトニアンシステム $(\mathrm{NHSAC})$ を導出する.さらに制御入力項と出力方程式 


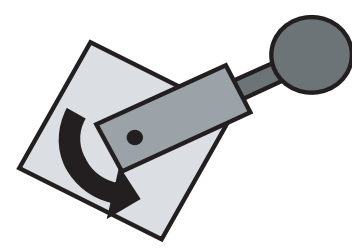

[a] Space Robot with Initial Momentum

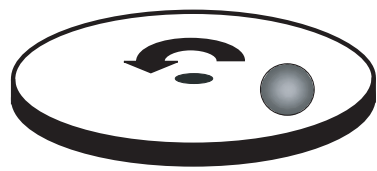

[c] Ball on Rotating Table

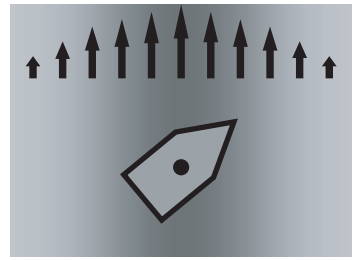

[b] Boat on Running River

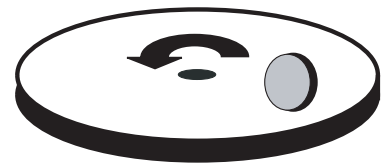

[d] Coin on Rotating Table
Fig. 1 Examples of systems with affine constraints

を加えた NHSAC の受動性についても考察する . そして 4.では, 本論文の結果を物理例である「回転盤上のコイ ン」に適用し，これまでの議論の検証を行う．

\section{2. 非ホロノミックアファイン拘束}

本章では, 本論文で扱うアファイン拘束について説 明を行う.システムの配位空間である $n$ 次元配位多 樣体を $Q$ とし，弚の一般化座標を $n$ 次元列ベクトル $q=\left[q_{1} \cdots q_{n}\right]^{\top} \in \mathbf{R}^{n}$ のように記述して，配位多樣体 の局所座標系と同一視して考える .また一般化速度を $\dot{q}=\left[\dot{q}_{1} \cdots \dot{q}_{n}\right]^{\top} \in \mathbf{R}^{n}$ で表す . 本論文では $n-m(m<n)$ 個の 1 階常微分方程式の組で表されるアファイン拘束

$$
A(q)+B(q) \dot{q}=0
$$

を考える .ここで $A(q) \in \mathbf{R}^{m}$ はアファイン項とよばれ， また $B(q) \in \mathbf{R}^{(n-m) \times n}$ である.アファイン拘束 (1) に関 して, 独立性の十分条件

$$
\operatorname{rank} B(q)=n-m, \forall q \in Q
$$

を仮定する．

つぎに,アファイン拘束の完全非ホロノミック性につい てまとめる.アファイン拘束 (1) は, 幾何学的に二つの組 $(D, X)$ で表すことができる $[8,10]$. ここで, $D$ は $(1)$ 式の $B(q)$ に対する $m$ 個の独立な零化べクトル $Y_{1}, \cdots, Y_{m}$ の 張る $Q$ 上の $m$ 次元分布，つまり $D:=\operatorname{span}\left\{Y_{1}, \cdots, Y_{m}\right\}$ である.また $X$ は成分に関して $A(q)+B(q) X(q)=0$ を満たす $Q$ 上のべクトル場であり，アファインベクト ル場とよばれる .これらのべクトル場 $X, Y_{1}, \cdots, Y_{m}$ の決 め方は一意ではないところに注意する。光して， $C_{0}$ を ベクトル場 $Y_{1}, \cdots, Y_{m}$ から成るすべてのLie括弧を含み， $\forall Z \in C_{0}$ について $[X, Z] \in C_{0}$ となるような最小のイン ボリューティブな分布とする.アファイン拘束が完全非 ホロノミックであるための必要十分条件は以下のように 与えられ [10]，本論文ではこの完全非ホロノミック性を 仮定する .
【定理 1】 $n$ 次元配位多樣体 $Q$ 上のアファイン拘束 (1) に対して以下の条件 (a), (b) は等価であり, これらが 成り立つとき，アファイン拘束は完全非ホロノミックま たは完全非可積分であるとよばれる .

(a) 第 1 積分が存在しない.

(b) 分布 $C_{0}$ に関して,

$$
\operatorname{dim} C_{0}(q)=n, \forall q \in Q
$$

が成り立つ。

最後に, アファイン変数・非アファイン变数を導入す る.一般化座標 $q$ を適当な並べ替えを行うことにより， $q=\left[q_{a}^{\top} q_{b}^{\top}\right]^{\top}$ のように二つに分ける .ここで, $q_{a} \in \mathbf{R}^{r}$ を アファイン変数, $q_{b} \in \mathbf{R}^{n-r}$ を非アファイン変数とよび, アファイン拘束 (1) を以下のように書き換える .

$$
A(q)+B_{a}(q) \dot{q}_{a}+B_{b}(q) \dot{q}_{b}=0
$$

ここで(4) 式に対して，以下の仮定を課すことにする .

（仮定 1） アファイン拘束（4)において，以下の (i)(iv)が成り立つ .

(i) アファイン拘束の数 $n-m$ とアファイン变数の次元 $r$ について, 以下が成り立つ。

$$
r \leq n-m
$$

(ii) アファイン項 $A$ は, 非アファイン变数 $q_{b}$ のみの関 数である.

(iii) (4) 式において, 非アファイン変数の速度を零 , つ まり $\dot{q}_{b}=0$ とした式

$$
A\left(q_{b}\right)+B_{a}(q) \dot{q}_{a}=0
$$

が物理的に成り立つ。

(iv) $B_{a} \in \mathbf{R}^{(n-m) \times r}$ は列フルランク ,つまり

$$
\operatorname{rank} B_{a}(q)=r, \forall q \in Q
$$

となる

仮定 1 (iii) は, 一般化座標のうち, 外部から入力を加 えなくても,アファイン項の影響によって变化が生じる変 数がアファイン变数, ということを意味し, システムの物 理的考察と上記の条件によって,一般化座標をアファイン 変数と非アファイン変数に一意的に分けることができる. その結果, アファインベクトル場はアファイン拘束の影 響を表し, アファイン変数へのみ変化を与えなければな らないので, $X=\left[X_{a}^{\top} X_{b}^{\top}\right]^{\top} \in \mathbf{R}^{n}, X_{a} \in \mathbf{R}^{r}, X_{b} \in \mathbf{R}^{n-r}$ とすると, $A+B_{a} X_{a}=0, X_{b}=0_{n-r}$ を満たさなければ ならず, 一般的にアファインベクトル場が

$$
X=\left[\begin{array}{c}
-B_{a}(q)^{\dagger} A\left(q_{b}\right) \\
0
\end{array}\right]
$$

のように一意に定まる.ここで $B_{a}^{\dagger}:=\left(B_{a}^{\top} B_{a}\right)^{-1} B_{a}^{\top}$ と おいた . 具体例は 4. を参照されたい . 
3. アファイン拘束を受ける非ホロノミッ

$$
\text { クハミルトニアンシステム }
$$

3.1 相空間上のハミルトニアンシステム

まず本節では , システムの設定ならびに相空間にお けるハミルトニアンシステムの概説を行う.シンプレ クティック多樣体・ポアソン多樣体・ハミルトンカ学 の詳細は $[15,16]$ などを参照されたい $Q Q$ を $n$ 次元配 位多樣体とし，弚の局所座標系である一般化座標を $q=\left[q^{1} \cdots q^{n}\right]^{\top} \in \mathbf{R}^{n}$ で表す. 乥して， $T^{*} Q$ を $Q$ の余接 バンドルとし，一般化運動量を $p=\left[p_{1} \cdots p_{n}\right]^{\top} \in \mathbf{R}^{n}$ と する . この一般化運動量 $p$ は基底 $d q^{k}(k=1, \cdots, n)$ を用 いて正準 1 形式

$$
\Theta:=\sum_{k=1}^{n} p_{k} d q^{k}
$$

で表されているところに注意する．すると， $T^{*} Q$ は相空 間とよばれる $2 n$ 次元多樣体であり，一般化座標と一般 化運動量の組 $(q, p)$ で記述される，乥して，正準 $\mathbf{2}$ 形式

$$
\Omega:=-d \Theta=\sum_{k=1}^{n} d q^{k} \wedge d p_{k}
$$

を定義すると，これは閉じており $(d$ を外微分演算子と すると $d \Omega=0)$ ，非退化で交代 2 形式であるので , 組 $\left(T^{*} Q, \Omega\right)$ はシンプレクティック多様体である.ゆえに， $T^{*} Q$ はポアソン多様体でもあるので, $T^{*} Q$ 上の任意の 関数 $f, g: T^{*} Q \rightarrow \mathbf{R}$ に対して,ポアソン括弧

$$
\{f, g\}:=\sum_{k=1}^{n}\left(\frac{\partial f}{\partial q^{k}} \frac{\partial g}{\partial p_{k}}-\frac{\partial f}{\partial p_{k}} \frac{\partial g}{\partial q^{k}}\right)
$$

が備わっている. 関数 $f, g, h: T^{*} Q \rightarrow \mathbf{R}$, 実定数 $a, b \in \mathbf{R}$ に対し，ポアソン括弧は(i) 歪対称性: $\{f, g\}=-\{g, f\}$, (ii) 双線形性 : $\{f, a g+b h\}=a\{f, g\}+b\{f, h\}$, (iii) ヤ コビの恒等式: $\{\{f, g\}, h\}=\{\{g, h\}, f\}+\{\{h, f\}, g\}$, (iv) ライプニッツの規則 : $\{f g, h\}=f\{g, h\}+g\{f, h\}$ を満たす.なお，ポアソン括弧 (11) は関数 $f, g$ の八

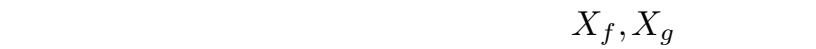
$\{f, g\}:=\Omega\left(X_{f}, X_{g}\right)$ のようにも定義することができる .

システムのハミルトニアンを時間変数 $t$ にも依存する 関数として $H(t, q, p)$ で表す.すると $T^{*} Q$ 上でハミル卜 ニアンシステム

$$
\left[\begin{array}{c}
\dot{q} \\
\dot{p}
\end{array}\right]=\underbrace{\left[\begin{array}{cc}
O_{n} & I_{n} \\
-I_{n} & O_{n}
\end{array}\right]}_{J}\left[\begin{array}{l}
\frac{\partial H^{\top}}{\partial q} \\
\frac{\partial H^{\top}}{\partial p}
\end{array}\right]
$$

が定義される .ここで, (12) 式の係数行列 $J$ はポアソン 構造行列とよばれ，これはポアソン括弧を用いて $(q, p)$ が正準座標であるという性質から，

$$
J=\left[\begin{array}{cc}
\left(\left\{q^{i}, q^{j}\right\}\right)_{i j} \\
\hdashline\left(\left\{p_{i}, q^{j}\right\}\right)_{i j} ! \\
\left.\left.\hdashline\left(\left\{p_{i}, p_{j}\right\}\right)_{i j}, p_{j}\right\}\right)_{i j}
\end{array}\right], \quad i, j=1, \cdots, n
$$

より導出されるところに注意する。

3.2 拡大相空間上のハミルトニアンシステム アファイン拘束 $(1)$ は, 微分 1 形式として ,

$$
A(q) d t+B(q) d q=0
$$

とも書くことができ, 時間変数 $t$ が陽に表れる.したがっ て , アファイン拘束を受けるシステムを余接バンドルで 導出する場合には, 時間変数 $t$ の空間 $\mathbf{R}$ をも含めた空間 $\mathbf{R} \times Q$ まで拡大して考えなければならないことがわかる. 弚こで本節では，時間変数も含めた拡大相空間を考え， 弚の上で拡大されたハミルトニアンシステムを考える．

まず時間変数 $t \in \mathbf{R}$ を $q^{0}:=t$ とおき, 時間も新し い変数の一つと考える (Fig. 2 (a)).さらに新しい時 間変数を $s \in \mathbf{R}$ と記述し，これは実際は $t$ と一致して いる. 時間変数を含めた配位多樣体を拡大配位多様体 とよび, $\bar{Q}:=\mathbf{R} \times Q$ と書く.$q^{0}$ に対応する運動量は 八ミルトニアン $H$ を用いて $, p_{0}:=-H$ で与えられる ことがわかっている $[4,6,7,16]$.つまり, $2 n+2$ 次元拡 大相空間 $T^{*} \bar{Q}$ の正準座標は $\bar{q}:=\left[q^{0} q^{1} \cdots q^{n}\right]^{\top} \in \mathbf{R}^{n+1}$ と $\bar{p}:=\left[p_{0} p_{1} \cdots p_{n}\right]^{\top} \in \mathbf{R}^{n+1}$ の組 $(\bar{q}, \bar{p})$ で与えられる (Fig. $2(\mathrm{~b}))$. この $\bar{p}$ は基底 $d q^{k}(k=0,1, \cdots, n)$ を用いて 拡大正準 1 形式

$$
\bar{\Theta}:=\sum_{k=0}^{n} p_{k} d q^{k}=p_{0} d q^{0}+\Theta
$$

で表される，光して，(10) 式と同樣に拡大正準 $\mathbf{2}$ 形式

$$
\bar{\Omega}:=-d \bar{\Theta}=\sum_{k=0}^{n} d q^{k} \wedge d p_{k}=d q^{0} \wedge d p_{0}+\Omega
$$

を定義すると，これもまた閉じた非退化で交代 2 形式で あるので, 組 $\left(T^{*} \bar{Q}, \bar{\Omega}\right)$ もシンプレクティック多樣体で ある.したがって， $T^{*} \bar{Q}$ もまたポアソン多樣体であるの で, $T^{*} \bar{Q}$ 上の任意の関数 $\bar{f}, \bar{g}: T^{*} \bar{Q} \rightarrow \mathbf{R}$ に対して, 拡大 ポアソン括弧

$$
\{\bar{f}, \bar{g}\}:=\sum_{k=0}^{n}\left(\frac{\partial \bar{f}}{\partial q^{k}} \frac{\partial \bar{g}}{\partial p_{k}}-\frac{\partial \bar{f}}{\partial p_{k}} \frac{\partial \bar{g}}{\partial q^{k}}\right)
$$

を用いることができる(簡略化のため $T^{*} Q$ 上のポアソ ン括弧 (11) と同じ記号を用いる) . 拡大ポアソン括弧も $T^{*} Q$ 上のポアソン括弧 (11) の性質 (i)-(iv) を同樣に満た すことが確認できる .

さらに $T^{*} \bar{Q}$ 上において拡大ハミルトニアン $\bar{H} を$

$$
\bar{H}\left(q^{0}, q, p_{0}, p\right):=H\left(q^{0}, q, p\right)+p_{0}
$$

のように定義すると， $T^{*} \bar{Q}$ 上で拡大ハミルトニアンシス テム 


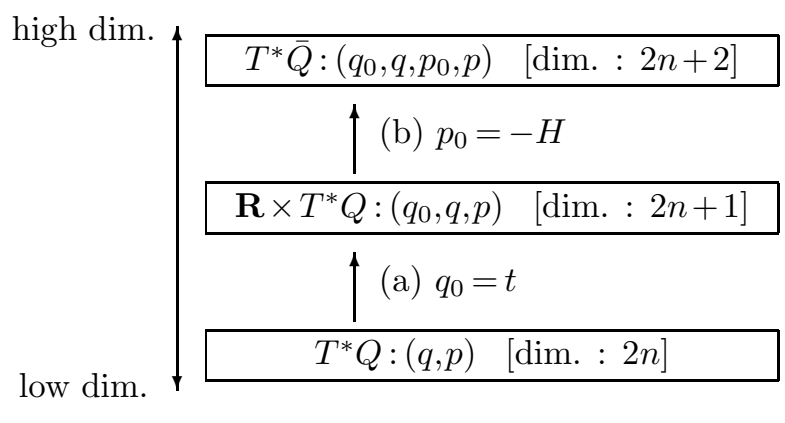

(c) Trans. (23)

$\begin{array}{r}\hline T^{*} \bar{Q}:\left(\tilde{q}_{0}, \tilde{q}, \tilde{p}_{0}, \tilde{p}\right) \quad[\operatorname{dim} .: 2 n+2] \\ \text { (d) } \tilde{q}_{0}=t, \frac{\partial \tilde{\tilde{H}}}{\partial \tilde{p}_{2}}=0_{n-m} \downarrow \\ \hline \mathcal{X}_{0}:\left(\tilde{q}, \tilde{p}_{0}, \tilde{p}_{1}\right)[\text { dim. }: n+m+1] \\ \text { (e) } \tilde{p}_{0}=-\tilde{H} \downarrow \\ \hline \mathcal{X}:\left(\tilde{q}, \tilde{p}_{1}\right) \quad[\text { dim. }: n+m] \\ \hline\end{array}$

Fig. 2 Diagram for derivation of NHSAC

$$
\left[\begin{array}{c}
q^{0^{\prime}} \\
q^{\prime} \\
p_{0}^{\prime} \\
p^{\prime}
\end{array}\right]=\underbrace{\left[\begin{array}{c:c:c:c}
0 & 0_{n} & 1 & 0_{n} \\
\hdashline 0_{n} & \bar{O}_{n} & 0_{n} & \bar{I}_{n} \\
\hdashline-1 & 0_{n} & 0 & 0_{n} \\
\hdashline 0_{n} & -I_{n} & 0_{n} & \bar{O}_{n}
\end{array}\right]}_{\bar{J}}\left[\begin{array}{c}
\frac{\partial \bar{H}}{\partial q^{0}} \\
\frac{\partial \bar{H}^{\top}}{\partial q} \\
\frac{\partial H}{\partial p_{0}} \\
\frac{\partial \bar{H}}{\partial p}
\end{array}\right]
$$

を定義することができる $[4,6,7,16]$. ここでダッシュ'は 時間変数 $s$ による微分を表している. (19) 式における拡 大ポアソン構造行列 $\bar{J}$ は拡大ポアソン括弧を用いて，

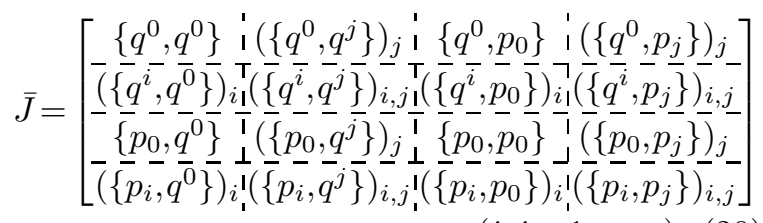

$$
(i, j=1, \cdots, n)
$$

より導出される . (20) 式の形からもわかるように , (19) 式は $T^{*} Q$ における通常のハミルトニアンシステム $(12)$ の $T^{*} \bar{Q}$ への自然な拡張になっている . (19) 式は $2 n+2$ 次元拡大相空間 $T^{*} \bar{Q}$ で記述されているが，定義より

$$
p_{0}=-H\left(q^{0}, q, p\right)
$$

という $2 n+1$ 次元の超曲面にシステムの運動が制限 されていることがわかる .つまり , システムの空間は $\mathbf{R} \times T^{*} Q$ で表され， $\left(q^{0}, q, p\right)$ で記述される．したがって， もしハミルトニアン $H$ が時間に依存しなければ $H$ は常 に定数であり, $p_{0}$ が定数の $2 n+1$ 次元超平面上を動くこ とになる . 拡大ハミルトニアン $(18)$ は钅の超曲面上では $p_{0}=-H$ であるので, 常に $\bar{H}=0$ となっていることにも 注意する .

\subsection{NHSAC の導出}

前節までの議論に基づいて , 本節ではアファイン拘束を 受ける非ホロノミックハミルトニアンシステム (NHSAC) を導出する．簡単のために，ハミルトニアンは時不変で ある $H(q, p)$ を考える . まず , システムがアファイン拘 束 (1) を受けるとすると , $\lambda \in \mathbf{R}^{n-m}$ をラグランジュ乗 数として (19) 式に拘束力の項を加えて，

$$
\left[\begin{array}{c}
q^{0 \prime} \\
q^{\prime} \\
p_{0}^{\prime} \\
p^{\prime}
\end{array}\right]=\underbrace{\left[\begin{array}{c:c:c:c}
0 & 0_{n} & 1 & 0_{n} \\
\hdashline 0_{n} & \bar{O}_{n} & 0_{n} & \bar{I}_{n} \\
\hdashline-1 & 0_{n} & 0 & 0_{n} \\
\hdashline 0_{n} & -I_{n} & 0_{n} & \bar{O}_{n}
\end{array}\right]}_{\bar{J}}\left[\begin{array}{c}
\frac{\partial \bar{H}}{\partial q^{0}} \\
\frac{\partial \bar{H}^{\top}}{\partial q} \\
\frac{\partial \bar{H}}{\partial p_{0}} \\
\frac{\partial \bar{H}^{\top}}{\partial p}
\end{array}\right]+\left[\begin{array}{c}
0 \\
0_{n} \\
A^{\top} \\
B^{\top}
\end{array}\right] \lambda
$$

となる．つぎに拡大相空間 $T^{*} \bar{Q}$ において, 以下の座 標変換 $\left(q^{0}, q, p_{0}, p\right) \rightarrow\left(\tilde{q}^{0}, \tilde{q}, \tilde{p}_{0}, \tilde{p}\right), \tilde{q}^{0} \in \mathbf{R}, \tilde{q} \in \mathbf{R}^{n}, \tilde{p}_{0} \in$ $\mathbf{R}, \tilde{p} \in \mathbf{R}^{n}$ を考える (Fig. 2 (c)).

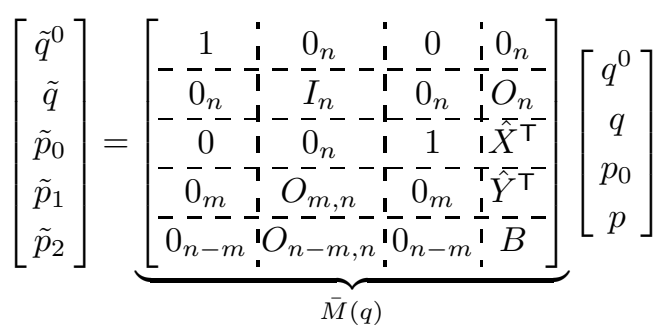

ここで, $\tilde{p}:=\left[\tilde{p}_{1}^{\top} \tilde{p}_{2}^{\top}\right]^{\top}, \tilde{p}_{1} \in \mathbf{R}^{m}, \tilde{p}_{2} \in \mathbf{R}^{n-m}$ と分割し, さらに $(23)$ 式による変換後の拡大ハミルトニアン $\bar{H}$ を $\tilde{\bar{H}}$ とすると , $\tilde{p}_{2}$ は

$$
\frac{\partial \tilde{\bar{H}}^{\top}}{\partial \tilde{p}_{2}}=0_{n-m}
$$

を満たすと仮定する．また，アファイン拘束に関するべク トル場を $\hat{X}, \hat{Y}_{1}, \cdots, \hat{Y}_{m}$ と記述し $, \hat{Y}(q):=\left[\hat{Y}_{1} \cdots \hat{Y}_{m}\right]$ と おく.ここで $\hat{Y}$ の定義により，(23) 式の変換行列 $\bar{M}(q)$ は常に正則となる . この座標変換 $(23)$ は後で見るように 拡大正準 2 形式 (16) を不変にしないので, 正準変換では ないところを留意されたい. (23) 式の逆変換は

$$
\left[\begin{array}{c}
q^{0} \\
q \\
p_{0} \\
p
\end{array}\right]=\underbrace{\left[\begin{array}{c:c:c:c:c}
1 & 0_{n} & 0 & 0_{m} & 0_{n-m} \\
\hdashline 0_{n} & \bar{I}_{n}-0_{n} & O_{n, m} & O_{n, n-m} \\
\hdashline 0 & 0_{n} & 1 & -\hat{X}^{\top} & U_{-}-\hat{X}^{\top} V \\
\hdashline 0_{m} & O_{m, n} ! 0_{m} & U & V
\end{array}\right]}_{M(\tilde{q})^{-1}}\left[\begin{array}{c}
\tilde{q}^{0} \\
\tilde{q} \\
\tilde{p}_{0} \\
\tilde{p}_{1} \\
\tilde{p}_{2}
\end{array}\right]
$$

で与えられ，ここで 


$$
[U V]:=\left[\begin{array}{c}
\hat{Y}^{\top} \\
B
\end{array}\right]^{-1}, U \in \mathbf{R}^{n \times m}, V \in \mathbf{R}^{n \times(n-m)}
$$

とおいている .

座標変換 (23) を用いると, 拡大ポアソン構造行列 (20) は計算によって，

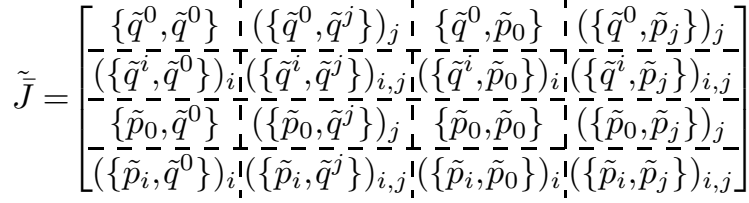

$$
\begin{aligned}
& (i, j=1, \cdots, n)
\end{aligned}
$$

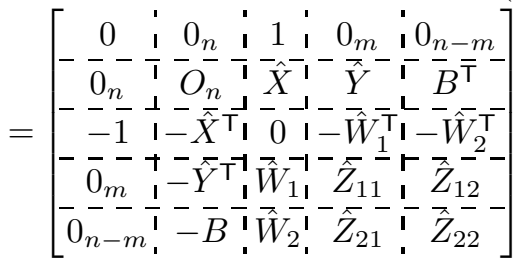

となることが確認できる .ここで $B$ の行ベクトルを $B_{1}, \cdots, B_{n-m}$ として ,

$$
\begin{aligned}
& \left(\hat{W}_{1}\right)_{i}:=\hat{Y}_{i}^{\top} \frac{\partial\left(\hat{X}^{\top} p\right)}{\partial q}-\frac{\partial\left(\hat{Y}_{i}^{\top} p\right)}{\partial q} \hat{X}, i=1, \cdots, m \\
& \left(\hat{W}_{2}\right)_{i}:=B_{i} \frac{\partial\left(\hat{X}^{\top} p\right)}{\partial q}-\frac{\partial\left(B_{i} p\right)}{\partial q} \hat{X}, i=m+1, \cdots, n \\
& \left(\hat{Z}_{11}\right)_{i, j}:=-p^{\top}\left[\hat{Y}_{i}, \hat{Y}_{j}\right], i, j=1, \cdots, m \\
& \left(\hat{Z}_{12}\right)_{i, j}:=-p^{\top}\left[\hat{Y}_{i}, B_{j}^{\top}\right], i=1, \cdots, m, j=1, \cdots, n-m \\
& \left(\hat{Z}_{21}\right)_{i, j}:=-\left(\hat{Z}_{12}\right)_{j, i}, i=1, \cdots, n-m, j=1, \cdots, m \\
& \left(\hat{Z}_{22}\right)_{i, j}:=-p^{\top}\left[B_{i}^{\top}, B_{j}^{\top}\right], i, j=1, \cdots, n-m
\end{aligned}
$$

とおいており ([·, , ] はリー括弧を表す $),$ とくに $\hat{Z}_{11}, \hat{Z}_{22}$ は定義より歪対称行列となる.拡大ハミルトニアン $\bar{H}$ は 座標変換 $(23)$ によって，

$$
\begin{aligned}
& \tilde{\bar{H}}\left(\tilde{q}^{0}, \tilde{q}, \tilde{p}_{0}, \tilde{p}\right) \\
& :=\bar{H}\left(\tilde{q}, \tilde{p}_{0}-\hat{X}^{\top}[U V] \tilde{p},[U V] \tilde{p}\right) \\
& =H(\tilde{q},[U V] \tilde{p})+\tilde{p}_{0}-\hat{X}^{\top}[U V] \tilde{p}
\end{aligned}
$$

に変換される．したがって，(22) 式は $(27),(29)$ 式を用 いて, $2 n+2$ 次元拡大相空間 $T^{*} \bar{Q}$ 上で

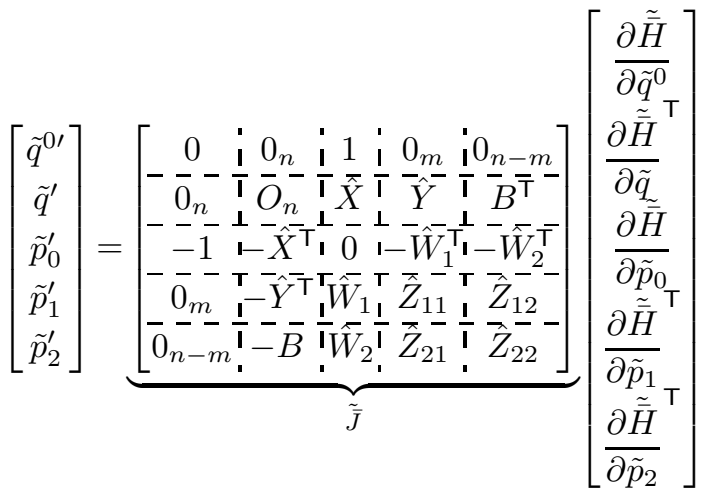

$$
+\left[\begin{array}{c}
0 \\
O_{n, n-m} \\
0 \\
O_{m, n-m} \\
B B^{\top}
\end{array}\right] \lambda
$$

と変換することができる．

ここで(30) 式の低次元化を行う . (30) 式の第 1 式は， $t$ と $s$ を同一視して $(23)$ 式を用いると，

$$
\frac{\partial \tilde{\bar{H}}}{\partial \tilde{p}_{0}}=\frac{\partial \tilde{q}^{0}}{\partial s}=\frac{\partial \tilde{q}^{0}}{\partial t}=\frac{\partial q^{0}}{\partial t}=1
$$

を表していることがわかるので, $\tilde{q}^{0}=t$ として $\tilde{q}^{0}$ の 1 次 元分を減らすことができる.なお，(31) 式は拡大八ミル トニアン $(29)$ に対して満たされていることに注意する そして $\tilde{p}_{2}$ は $(24)$ 式を満たすので, $(22)$ 式の第 5 式は除 外することができ, $\tilde{p}_{2}$ の $n-m$ 次元分も減らすことがで きる．したがって，( $\left(\tilde{q}, \tilde{p}_{0}, \tilde{p}_{1}\right) て ゙$ 記述される $n+m+1$ 次元空間 $\mathcal{X}_{0}$ を

$$
\begin{aligned}
\mathcal{X}_{0}:= & \left\{\left(q, p^{0}, p\right) \in \mathbf{R} \times T^{*} Q \mid\right. \\
& \left.A(q) \frac{\partial H(t, q, p)}{\partial p_{0}}+B(q) \frac{\partial H(t, q, p)}{\partial p}=0\right\}
\end{aligned}
$$

のように記述すると (Fig. $2(\mathrm{~d})), T^{*} \bar{Q}$ 上の (30) 式は $\mathcal{X}_{0}$ 上て変数 $\left(\tilde{q}, \tilde{p}_{0}, \tilde{p}_{1}\right)$ を用いて，

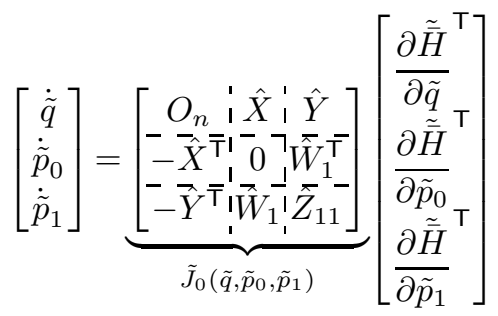

のように表すことができる. 光して，この $\mathcal{X}_{0}$ 上でもポ アソン括弧

$$
\{\tilde{f}, \tilde{g}\}:=\left[\frac{\partial \tilde{f}}{\partial \tilde{q}} \frac{\partial \tilde{f}}{\partial \tilde{p}_{0}} \frac{\partial \tilde{f}}{\partial \tilde{p}_{1}}\right] \tilde{J}_{0}\left(\tilde{q}, \tilde{p}_{0}, \tilde{p}_{1}\right)\left[\begin{array}{l}
\frac{\partial \tilde{g}^{\top}}{\partial \tilde{q}} \\
\frac{\partial \tilde{g}}{\partial \tilde{p}_{0}} \\
\frac{\partial \tilde{g}}{\partial \tilde{p}_{1}}
\end{array}\right]
$$

を定義することができ， $T^{*} Q$ 上のポアソン括弧の性質 (i) 歪対称性, (ii) 双線形性, (iv) ライプニッツの規則を 満たすことが確認できる．しかし，(iii) ヤコビの恒等式 は必ずしも成り立たない，ポアソン多樣体は，性質 (i), (ii), (iv) のみを満たす場合は特に概ポアソン多様体とよ ばれ，非ホロノミックシステムを考える場合において重 要な役割を果たす $[8]$.

乥して，さらなる超曲面への低次元化を考える．座標 変換 $(23)$ により, $T^{*} \bar{Q}$ の超曲面 $(21)$ は 


$$
\tilde{p}_{0}=-H(\tilde{q},[U V] \tilde{p})+\hat{X}^{\top}[U V] \tilde{p}=:-\tilde{H}(\tilde{q}, \tilde{p})(35)
$$

となり， $\tilde{p}_{0}$ が分離されているままであることがわかる .

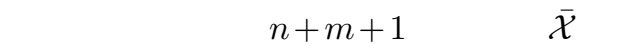

$$
\tilde{p}_{0}=-\tilde{H}\left(\tilde{q}, \tilde{p}_{1}\right)
$$

とおいて , 変数を $\left(\tilde{q}, \tilde{p}_{1}\right)$ としてシステムを考えることに よって，さらに $\tilde{p}_{0}$ の 1 次元分を減らすことができる .こ のとき, システムのハミルトニアンは (36) 式で定義され る $\tilde{H}$ となる . 以上より， $\left(\tilde{q}, \tilde{p}_{1}\right)$ て記述される $n+m$ 次元 空間 $\mathcal{X}_{0}$ を

$$
\mathcal{X}:=\left\{(q, p) \in T^{*} Q \mid A(q)+B(q) \frac{\partial H(q, p)}{\partial p}=0\right\}
$$

のように定義すると $($ Fig. $2(\mathrm{e})), T^{*} \bar{Q}$ 上の $(30)$ 式は $\mathcal{X}$ 上で以下のように表すことができる.

【定理 2】(37)式で定義される $n+m$ 次元空間 $\mathcal{X}$ 上 て変数を $\tilde{q} \in \mathbf{R}^{n}, \tilde{p}_{1} \in \mathbf{R}^{m}$ として, アファイン拘束を受け る非ホロノミックハミルトニアンシステム (Nonholonomic Hamiltonian Systems with Affine Constriants, NHSAC)は,

$$
\begin{aligned}
& {\left[\begin{array}{c}
\dot{\tilde{q}} \\
\dot{\tilde{p}}_{1}
\end{array}\right]=\left[\begin{array}{c}
\hat{X}(\tilde{q}) \\
\hat{W}_{1}\left(\tilde{q}, \tilde{p}_{1}\right)
\end{array}\right]} \\
& +\underbrace{\left[\begin{array}{cc}
O_{n} & \hat{Y}(\tilde{q}) \\
-\hat{Y}(\tilde{q})^{\top} \hat{Z}_{11}\left(\tilde{q}, \tilde{p}_{1}\right)
\end{array}\right]}_{\tilde{J}\left(\tilde{q}, \tilde{p}_{1}\right)}\left[\begin{array}{c}
\frac{\partial \tilde{H}^{\top}}{\partial \tilde{q}} \\
\frac{\partial \tilde{H}}{\partial \tilde{p}_{1}}
\end{array}\right]
\end{aligned}
$$

のように表される.ここで, ハミルトニアン $\tilde{H}$ は $(35)$ 式， $\hat{W}_{1}, \hat{Z}_{11}$ は (28) 式で光れ光れ定義され，これらはX 上で $\tilde{q}$ と $\tilde{p}_{1}$ の関数で表される.

線形拘束の場合のハミルトニアンシステム $[3]$ と比べ， NHSAC (38) にはアファイン拘束に起因する右辺第 1 項 が存在するところが大きな違いである .なおアファイ ン拘束に関して $A \equiv 0 つ ま り ~ \hat{X} \equiv 0$ とおくことにより， NHSAC (38) は線形拘束の場合のハミルトニアンシステ ム $[3]$ と一致するところに注意されたい．また，ハミル トニアンの中の慣性行列 $G$ が単位行列の簡単な場合を考 えると, $(37)$ 式は $\mathcal{X}=\left\{(\tilde{q}, \tilde{p}) \mid \tilde{p}_{2}=-A(\tilde{q})\right\}$ と表すこと ができる .これは [3] で示されている線形拘束の場合に おける座標変換後の拘束空間 $\mathcal{X}=\left\{(\tilde{q}, \tilde{p}) \mid \tilde{p}_{2}=0\right\}$ とも 一致する .

\subsection{NHSAC の受動性}

最後に本節では，NHSAC の受動性について考察す る.NHSAC (38) には制御入力が含まれていないが, 元 のシステム $(22)$ の第 4 式に制御入力項 $E(q) u, E(q) \in$ $\mathbf{R}^{n \times m}, u \in \mathbf{R}^{m}$ を加えた場合を考えると，NHSAC (38) は以下のように得られることがわかる .

$$
\left[\begin{array}{c}
\dot{\tilde{q}} \\
\tilde{\tilde{p}}_{1}
\end{array}\right]=\left[\begin{array}{c}
\hat{X} \\
\hat{W}_{1}
\end{array}\right]+\left[\begin{array}{cc}
O_{n} & \hat{Y} \\
-\hat{Y}^{\top} & \hat{Z}_{11}
\end{array}\right]\left[\begin{array}{c}
\frac{\partial \tilde{H}^{\top}}{\partial \tilde{q}} \\
\frac{\partial \tilde{H}^{\top}}{\partial \tilde{p}_{1}}
\end{array}\right]+\left[\begin{array}{c}
0_{n} \\
\hat{Y}^{\top} E
\end{array}\right] u
$$

また，出力 $y \in \mathbf{R}^{m}$ を

$$
y=E^{\top} \hat{Y}{\frac{\partial \tilde{H}^{\top}}{\partial \tilde{p}_{1}}}^{\top}
$$

のように設定する .一般的にハミルトニアンシステムに 対して, ハミルトニアン $\tilde{H}$ が準正定関数であり, さらに

$$
\dot{\tilde{H}}=\frac{d \tilde{H}}{d t} \leq y^{\top} u
$$

が満たされるとき, 受動性を満たすとよばれる．以下の 定理は, NHSAC (39), (40)が受動性を満たすための条 件を与える。

【定理 3】 NHSAC (39), (40)が受動性を満たすため の必要十分条件は, $\tilde{H}$ が準正定関数であり, かつ

$$
\frac{\partial \tilde{H}\left(\tilde{q}, \tilde{p}_{1}\right)}{\partial \tilde{q}} \hat{X}(\tilde{q})+\frac{\partial \tilde{H}\left(\tilde{q}, \tilde{p}_{1}\right)}{\partial \tilde{p}_{1}} \hat{W}_{1}\left(\tilde{q}, \tilde{p}_{1}\right) \leq 0
$$

が成り立つことである

(証明) ハミルトニアン $\tilde{H}$ NHSAC (39), (40)の解 軌道上において時間 $t$ で微分すると，

$$
\begin{aligned}
& \dot{\tilde{H}}=\frac{\partial \tilde{H}}{\partial \tilde{q}} \dot{\tilde{q}}+\frac{\partial \tilde{H}}{\partial \tilde{p}_{1}} \dot{\tilde{p}}_{1} \\
& =\left[\frac{\partial \tilde{H}}{\partial \tilde{q}} \frac{\partial \tilde{H}}{\partial \tilde{p}_{1}}\right] \\
& \times\{\left[\begin{array}{c}
\hat{X} \\
\hat{W}_{1}
\end{array}\right]+\underbrace{\left[\begin{array}{cc}
O_{n} & \hat{Y} \\
-\hat{Y}^{\top} & \hat{Z}_{11}
\end{array}\right]}_{\tilde{J}}\left[\begin{array}{c}
\frac{\partial \tilde{H}^{\top}}{\partial \tilde{q}} \\
\frac{\partial \tilde{H}^{\top}}{\partial \tilde{p}_{1}}
\end{array}\right]+\left[\begin{array}{c}
0_{n} \\
\hat{Y}^{\top} E
\end{array}\right] u\} \\
& =\frac{\partial \tilde{H}}{\partial \tilde{q}} \hat{X}+\frac{\partial \tilde{H}}{\partial \tilde{p}_{1}} \hat{W}_{1}+\frac{\partial \tilde{H}^{\top}}{\partial \tilde{p}_{1}} \hat{Y}^{\top} E u \\
& =\frac{\partial \tilde{H}}{\partial \tilde{q}} \hat{X}+\frac{\partial \tilde{H}}{\partial \tilde{p}_{1}} \hat{W}_{1}+y^{\top} u
\end{aligned}
$$

となる．ここでJが歪対称行列という性質を使っている． したがって，題意が示された .

定理 3 の証明では , ハミルトニアン $\tilde{H}$ の時間微分を陽に 計算しているが, 実は, 元の拡大ハミルトニアンシステ ム $(30)$ の第 3 式において,$\tilde{p}_{0}=-\tilde{H}$ を代入しても $(42)$ 式と同じ結果が得られる．つまり，(30) 式の第 3 式はシ ステムのハミルトニアンの時間変化を表している.線形 拘束の場合 , ハミルトニアンシステムの受動性は常に満 たされることは知られているが [9]，弚れは (42) 式にお いて $\hat{X} \equiv 0$ とおくことからも確かめられ，アファイン拘 束の影響がシステムの受動性を常に満たさなくさせてい ることがわかる . 


\section{4. 物理例}

本章では具体的な物理例として「回転盤上のコイ ン」[12-14] を取りあげる.Fig. 3 のように，一定角速 度で回転する回転盤の上にコインを乗せるような仮想 的なシステムを考える．ここでコインは回転盤と点で 接しているとし，倒れることなく鉛直に保ったまま水 平な回転盤の上を滑ることなく運動する場合を考える． 回転盤の中心が原点となるように $x y$ 座標系を取り，コ インが回転盤と接している位置を $(x, y)$ で表す．关の 接点を通り，コインの回転方向に引いた直線が $x$ 軸の 正の方向と成す角を $\theta$ とする . またコインの回転角を $\phi$ とする .これらにより, 回転盤上のコインの一般化

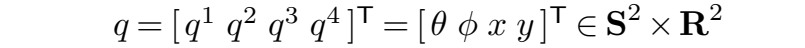
述され， $n=4$ である. $q$ に対応する一般化運動量を $p=\left[\begin{array}{lll}p_{1} & p_{2} & p_{3} \\ p_{4}\end{array}\right]^{\top}$ とおく.パラメータは回転盤の回転角 速度を $\Omega$, コインの質量を $M$, コインの半径を $R$, コイ ンの $\theta$ 方向， $\phi$ 方向の慣性モーメントを乥れ羿れ $J_{1}, J_{2}$ とする . すると , このシステムのハミルトニアンは

$$
H(q, p)=\frac{1}{2 J_{1}} p_{1}^{2}+\frac{1}{2 J_{2}} p_{2}^{2}+\frac{1}{2 M}\left(p_{3}^{2}+p_{4}^{2}\right)
$$

で与えられる .

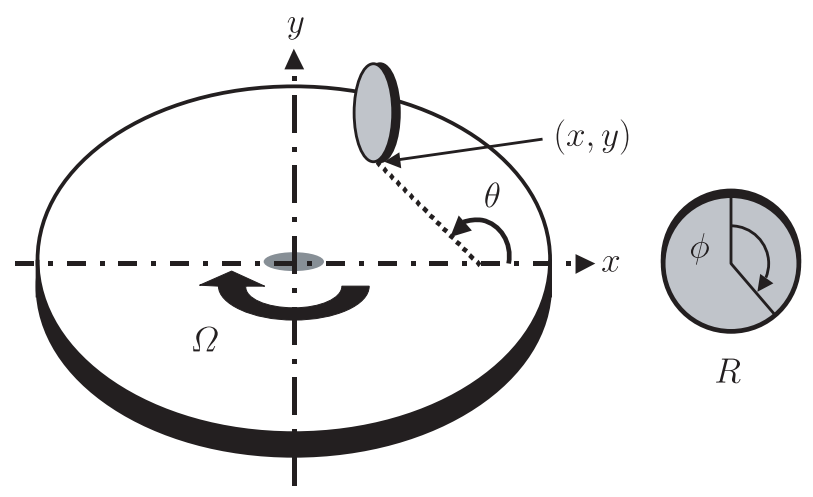

Fig. 3 Coin on rotating table

つぎに , コインの回転方向・側面方向の速度の釣り合 いを考えることにより，このシステムが受けるアファイ ン拘束は

$$
\begin{gathered}
\underbrace{\left[\begin{array}{c}
0 \\
\Omega(y \cos \theta-x \sin \theta)
\end{array}\right]}_{A(q)} \\
+\underbrace{\left[\begin{array}{cccc}
0 & 0 & \sin \theta & -\cos \theta \\
0 & -R \cos \theta & \sin \theta
\end{array}\right]}_{B(q)}\left[\begin{array}{c}
\dot{\theta} \\
\dot{\phi} \\
\dot{x} \\
\dot{y}
\end{array}\right]=0
\end{gathered}
$$

で与えられ， $m=2$ である .このアファイン拘束 $(43)$ は 分布 $C_{0}$ を計算することにより，定理 1 から完全非ホ口 ノミックであることが確かめられる．コインは回転盤か ら角速度を与えられ自転するので, アファイン変数は $\phi$ となり，仮定 1 を満たす．ゆえに $\hat{X}, \hat{Y}$ は，

$$
\hat{X}=\left[\begin{array}{c}
0 \\
\frac{\Omega}{R}\left(q_{3} \sin q_{1}-q_{4} \cos q_{1}\right) \\
0 \\
0
\end{array}\right], \hat{Y}=\left[\begin{array}{cc}
1 & 0 \\
0 & 1 \\
0 & R \cos q_{1} \\
0 & R \sin q_{1}
\end{array}\right]
$$

のように求められる.

簡単のために ,パラメータに関して $M=R=J_{1}=J_{2}=$ 1 とおき ,変数を $\tilde{q}=\left[\tilde{q}^{1} \tilde{q}^{2} \tilde{q}^{3} \tilde{q}^{4}\right]^{\top} \in \mathbf{R}^{4}, \tilde{p}_{1}=\left[\tilde{p}_{11} \tilde{p}_{12}\right]^{\top} \in$ $\mathbf{R}^{2}$ と記述する.乥して，コインの $\theta, \phi$ 方向へのトルクを光 れ光れ制御入力 $u_{1}, u_{2}$ とし $, u=\left[u_{1} u_{2}\right]^{\top} \in \mathbf{R}^{2}, E(q):=$ $\left[\begin{array}{ll}I_{2} & O_{2,4}\end{array}\right]^{\top}$ とする.すると，計算によって NHSAC は (45), (46) 式で与えられることがわかり，ハミルトニア ンは (47)式のように表される .

最後に回転盤上のコインに対する NHSAC の受動性に ついて考える . 定理 3 の受動性条件 (42) を具体的に計算 すると ,

$$
\begin{aligned}
& -\frac{\Omega}{R^{3}+R}\left(\tilde{q}_{3} \cos \tilde{q}_{1}+\tilde{q}_{4} \sin \tilde{q}_{1}\right) \\
& \quad \times\left\{\tilde{p}_{12}+2 \Omega R\left(\tilde{q}_{3} \sin \tilde{q}_{1}-\tilde{q}_{4} \cos \tilde{q}_{1}\right)\right\} \tilde{p}_{11} \leq 0
\end{aligned}
$$

となる . (48)式が満たされていれば, NHSAC は受動性 を満たすことになる．もし回転盤が静止している場合， つまり平面上の転がるコインを考えるならば，(48)式に おいて $\Omega=0$ とすると左辺が恒等的に 0 となるので, 受 動性条件を常に満たしていることが確認できる．

\section{5. おわりに}

本論文では，拡大相空間における拡大ハミルトニア ンに対して座標変換と低次元化を行うことによって，ア ファイン拘束を受ける非ホロノミックハミルトニアンシ ステム $(\mathrm{NHSAC})$ を導出した . さらに制御入力項と出力 方程式を加えた NHSACに対して，受動性の条件を求め た . NHSAC は従来の線形拘束の場合を包含する，より 広いクラスのハミルトニアンシステムとなる .

今後の課題としては, 受動性条件を満たすような軌道 計画, NHSACに対する一般化正準変換則の提案, 受動 性に基づいた制御則の導出などが挙げられる。

\section{参 考文 献}

[1] A. M. Bloch, M. Reyhanoglu and H. McClamroch: Control and stabilization of nonholonomic dynamic systems; IEEE Trans. on Automat. Contr., Vol. 37, No. 11, pp. 1746-1757 (1992)

[2] R. M. Murray and S. S. Sastry: Nonholonomic motion planning: Steering using sinusoids; IEEE Trans. on Automatic Control, Vol. 38, No. 5, pp. 700-716 (1993)

[3] A. J. van der Schaft and B. M. Maschke: On the Hamiltonian formulation of nonholonomic mechanical systems; Reports on Mathematical Physics, 


$$
\begin{aligned}
& {\left[\begin{array}{c}
\dot{\tilde{q}}_{1} \\
\dot{\tilde{q}}_{2} \\
\dot{\tilde{q}}_{3} \\
\dot{\tilde{q}}_{4} \\
\dot{\tilde{p}}_{11} \\
\dot{\tilde{p}}_{12}
\end{array}\right]=\left[\begin{array}{c}
0 \\
\frac{\Omega}{R}\left(\tilde{q}_{3} \sin \tilde{q}_{1}-\tilde{q}_{4} \cos \tilde{q}_{1}\right) \\
0 \\
0 \\
-\frac{\Omega}{R^{3}+R}\left(\tilde{q}_{3} \cos \tilde{q}_{1}+\tilde{q}_{4} \sin \tilde{q}_{1}\right)\left\{\tilde{p}_{12}+2 \Omega R\left(\tilde{q}_{3} \sin \tilde{q}_{1}-\tilde{q}_{4} \cos \tilde{q}_{1}\right)\right\} \\
0
\end{array}\right]} \\
& +\left[\begin{array}{cccccc}
0 & 0 & 0 & 0 & 1 & 0 \\
0 & 0 & 0 & 0 & 0 & 1 \\
0 & 0 & 0 & 0 & 0 & R \cos \tilde{q}_{1} \\
0 & 0 & 0 & 0 & 0 & R \sin \tilde{q}_{1} \\
-1 & 0 & 0 & 0 & 0 & 0 \\
0 & -1 & -R \cos \tilde{q}_{1}-R \sin \tilde{q}_{1} & 0 & 0
\end{array}\right]\left[\begin{array}{c}
\frac{\partial \tilde{H}^{\top}}{\partial \tilde{q}} \\
\frac{\partial \tilde{H}^{\top}}{\partial \tilde{p}_{1}}
\end{array}\right]+\left[\begin{array}{ll}
0 & 0 \\
0 & 0 \\
0 & 0 \\
0 & 0 \\
1 & 0 \\
0 & 1
\end{array}\right] u \\
& {\left[\begin{array}{l}
y_{1} \\
y_{2}
\end{array}\right]=\left[\begin{array}{ll}
1 & 0 \\
0 & 1
\end{array}\right] \frac{\partial \tilde{H}^{\top}}{\partial \tilde{p}_{1}}} \\
& \tilde{H}\left(\tilde{q}, \tilde{p}_{1}\right)=\frac{1}{2} \tilde{p}_{11}^{2}+\frac{1}{2\left(R^{2}+1\right)} \tilde{p}_{12}^{2}-\frac{\Omega}{R^{3}+R}\left(\tilde{q}_{3} \sin \tilde{q}_{1}-\tilde{q}_{4} \cos \tilde{q}_{1}\right) \tilde{p}_{12}-\frac{\Omega^{2}}{2\left(R^{2}+1\right)}\left(\tilde{q}_{3} \sin \tilde{q}_{1}-\tilde{q}_{4} \cos \tilde{q}_{1}\right)^{2}
\end{aligned}
$$

Vol. 34, No. 2, pp. 225-233 (1994)

[4] A. V. Tsiganov: Canonical transformations of the extended phase space, Toda lattices an the Stäckel family of integrable systems; J. Physics A: Mathematical and General, Vol. 33, No. 22, pp. 4169-4182 (2000)

[5] K. Fujimoto and T. Sugie: Stabilization of Hamiltonian systems with nonholonomic constraints based on time-varying generalized canonical transformations; Systems \& Control Letters, Vol. 44, No. 4, pp. 309 319 (2001)

[6] J. Struckmeier: Hamiltonian dynamics on the symplectic extended phase space for autonomous and non-autonomous systems; J. Physics A: Mathematical and General, Vol. 38, No. 6, pp. 1257-1278 (2005)

[7] Y. Minesaki and Y. Nakamura: New numerical integrator for the Stäckel system conserving the same number of constants of motion as the degree of freedom; J. Physics A: Mathematical and General, Vol. 39, No. 30, pp. 9453-9476 (2006)

[8] A. M. Bloch: Nonholonomic Mechanics and Control, Springer-Verlag (2003)

[9] 藤本 ,杉江: 一般化正準変換を用いたあるクラスの非ホ口 ノミック系の安定化; 計測自動制御学会論文集, Vol. 36, No. 9, pp. 749-756 (2001)

[10] 甲斐，木村: 配位多樣体におけるアファイン拘束の理論 的解析 - Part I : アファイン拘束の可積分性・非可積分 条件と配位多樣体の葉層構造 - ; 計測自動制御学会論文 集, Vol. 42, No. 3, pp. 212-221 (2006)

[11] 甲斐，木村: 配位多樣体におけるアファイン拘束の理論 的解析 - Part II : アファイン拘束を受ける運動学的非
対称アファインシステムの可到達性 - ; 計測自動制御学 会論文集, Vol. 42, No. 3, pp. 222-231 (2006)

[12] T. Kai, H. Kimura and S. Hara: Nonlinear control analysis on kinematically asymmetrically affine control systems with affine constraints; Proc. of 16th IFAC World Congress (2005)

[13] T. Kai, H. Kimura and S. Hara: Nonlinear control analysis on nonholonomic dynamic systems with affine constraints; Proc. of 44th IEEE CDC-ECC, pp. 1459-1464 (2005)

[14] T. Kai: Affine constraints in nonlinear control theory; Proc. of 3rd Workshop on LHMNLC, pp. 251-256 (2006)

[15] 木村, 菅野: 微分形式による解析力学, 吉岡書店 (1996)

[16] 山本 , 中村: 解析力学 I, II, 朝倉書店 (1998)

[17] 美多: 非線形制御入門 - 劣駆動ロボットの技能制御論, 昭晃堂 (2000)

\section{著者略歴}

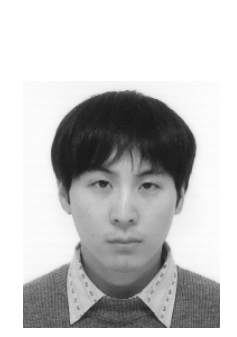
也 (正会員)

1976 年 11 月 5 日生. 2005 年東京大学大 学院新領域創成科学研究科博士課程修了. 同年大阪大学大学院工学研究科助手, 2007 年同大学同研究科助教, 現在に至る. 非線 形制御理論, 非ホロノミックシステムなど の研究に従事 . 博士 (科学) . 2004 年度計 測自動制御学会学術奨励賞, 2006 年度計測自動制御学会制御 部門大会賞，2007 年度計測自動制御学会論文賞受賞. 計測自 動制御学会，電子情報通信学会の会員. 